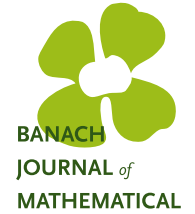

ANALYSIS
Banach J. Math. Anal. 10 (2016), no. 4, 864-897

http://dx.doi.org/10.1215/17358787-3649788

ISSN: $1735-8787$ (electronic)

http://projecteuclid.org/bjma

\title{
CONVEX CONES OF GENERALIZED MULTIPLY MONOTONE FUNCTIONS AND THE DUAL CONES
}

\author{
IOSIF PINELIS
}

Communicated by D. Yang

\begin{abstract}
Let $n$ and $k$ be nonnegative integers such that $1 \leq k \leq n+1$. The convex cone $\mathscr{F}_{+}^{k: n}$ of all functions $f$ on an arbitrary interval $I \subseteq \mathbb{R}$ whose derivatives $f^{(j)}$ of orders $j=k-1, \ldots, n$ are nondecreasing is characterized. A simple description of the convex cone dual to $\mathscr{F}_{+}^{k: n}$ is given. In particular, these results are useful in, and were motivated by, applications in probability. In fact, the results are obtained in a more general setting with certain generalized derivatives of $f$ of the $j$ th order in place of $f^{(j)}$. Somewhat similar results were previously obtained, in terms of Tchebycheff-Markov systems, in the case when the left endpoint of the interval $I$ is finite, with certain additional integrability conditions; such conditions fail to hold in the mentioned applications. Development of substantially new methods was needed to overcome the difficulties.
\end{abstract}

\section{INTRODUCTION}

In applications in probability (see, e.g., [11], [5], [6], [20], [21], [3], [26], [23], [25], and references therein), one is concerned with stochastic domination, defined by a formula of the form

$$
X \succcurlyeq Y \stackrel{\mathscr{F}}{\Longleftrightarrow} X \succcurlyeq Y(\bmod \mathscr{F}) \Longleftrightarrow \mathrm{def} f(X) \geq \mathrm{E} f(Y) \text { for all } f \in \mathscr{F},
$$

Copyright 2016 by the Tusi Mathematical Research Group.

Received Jul. 22, 2015; Accepted Feb. 1, 2016.

2010 Mathematics Subject Classification. Primary 46N10; Secondary 26A48, 26A51, 26A46, 26D05, 26D07, 26D10, 26D15, 34L30, 41A10, 46A55, 49K30, 49M29, 52A07, 52A41, 60E15, 90C25, 90C46.

Keywords. dual cones, multiply monotone functions, generalized moments, stochastic orders, probability inequalities. 


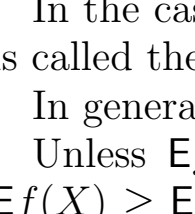

ANALYSIS
Banach J. Math. Anal. 10 (2016), no. 4, 864-897

http://dx.doi.org/10.1215/17358787-3649788

ISSN: $1735-8787$ (electronic)

http://projecteuclid.org/bjma

\title{
CONVEX CONES OF GENERALIZED MULTIPLY MONOTONE FUNCTIONS AND THE DUAL CONES
}

\author{
IOSIF PINELIS
}

Communicated by D. Yang

\begin{abstract}
Let $n$ and $k$ be nonnegative integers such that $1 \leq k \leq n+1$. The convex cone $\mathscr{F}_{+}^{k: n}$ of all functions $f$ on an arbitrary interval $I \subseteq \mathbb{R}$ whose derivatives $f^{(j)}$ of orders $j=k-1, \ldots, n$ are nondecreasing is characterized. A simple description of the convex cone dual to $\mathscr{F}_{+}^{k: n}$ is given. In particular, these results are useful in, and were motivated by, applications in probability. In fact, the results are obtained in a more general setting with certain generalized derivatives of $f$ of the $j$ th order in place of $f^{(j)}$. Somewhat similar results were previously obtained, in terms of Tchebycheff-Markov systems, in the case when the left endpoint of the interval $I$ is finite, with certain additional integrability conditions; such conditions fail to hold in the mentioned applications. Development of substantially new methods was needed to overcome the difficulties.
\end{abstract}

\section{INTRODUCTION}

In applications in probability (see, e.g., [11], [5], [6], [20], [21], [3], [26], [23], [25], and references therein), one is concerned with stochastic domination, defined by a formula of the form

$$
X \succcurlyeq Y \stackrel{\mathscr{F}}{\Longleftrightarrow} X \succcurlyeq Y(\bmod \mathscr{F}) \Longleftrightarrow \mathrm{def} f(X) \geq \mathrm{E} f(Y) \text { for all } f \in \mathscr{F},
$$

Copyright 2016 by the Tusi Mathematical Research Group.

Received Jul. 22, 2015; Accepted Feb. 1, 2016.

2010 Mathematics Subject Classification. Primary 46N10; Secondary 26A48, 26A51, 26A46, 26D05, 26D07, 26D10, 26D15, 34L30, 41A10, 46A55, 49K30, 49M29, 52A07, 52A41, 60E15, 90C25, 90C46.

Keywords. dual cones, multiply monotone functions, generalized moments, stochastic orders, probability inequalities. 


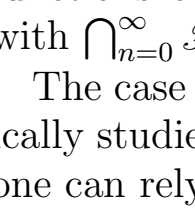

ANALYSIS
Banach J. Math. Anal. 10 (2016), no. 4, 864-897

http://dx.doi.org/10.1215/17358787-3649788

ISSN: $1735-8787$ (electronic)

http://projecteuclid.org/bjma

\title{
CONVEX CONES OF GENERALIZED MULTIPLY MONOTONE FUNCTIONS AND THE DUAL CONES
}

\author{
IOSIF PINELIS
}

Communicated by D. Yang

\begin{abstract}
Let $n$ and $k$ be nonnegative integers such that $1 \leq k \leq n+1$. The convex cone $\mathscr{F}_{+}^{k: n}$ of all functions $f$ on an arbitrary interval $I \subseteq \mathbb{R}$ whose derivatives $f^{(j)}$ of orders $j=k-1, \ldots, n$ are nondecreasing is characterized. A simple description of the convex cone dual to $\mathscr{F}_{+}^{k: n}$ is given. In particular, these results are useful in, and were motivated by, applications in probability. In fact, the results are obtained in a more general setting with certain generalized derivatives of $f$ of the $j$ th order in place of $f^{(j)}$. Somewhat similar results were previously obtained, in terms of Tchebycheff-Markov systems, in the case when the left endpoint of the interval $I$ is finite, with certain additional integrability conditions; such conditions fail to hold in the mentioned applications. Development of substantially new methods was needed to overcome the difficulties.
\end{abstract}

\section{INTRODUCTION}

In applications in probability (see, e.g., [11], [5], [6], [20], [21], [3], [26], [23], [25], and references therein), one is concerned with stochastic domination, defined by a formula of the form

$$
X \succcurlyeq Y \stackrel{\mathscr{F}}{\Longleftrightarrow} X \succcurlyeq Y(\bmod \mathscr{F}) \Longleftrightarrow \mathrm{def} f(X) \geq \mathrm{E} f(Y) \text { for all } f \in \mathscr{F},
$$

Copyright 2016 by the Tusi Mathematical Research Group.

Received Jul. 22, 2015; Accepted Feb. 1, 2016.

2010 Mathematics Subject Classification. Primary 46N10; Secondary 26A48, 26A51, 26A46, 26D05, 26D07, 26D10, 26D15, 34L30, 41A10, 46A55, 49K30, 49M29, 52A07, 52A41, 60E15, 90C25, 90C46.

Keywords. dual cones, multiply monotone functions, generalized moments, stochastic orders, probability inequalities. 


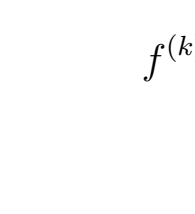

ANALYSIS
Banach J. Math. Anal. 10 (2016), no. 4, 864-897

http://dx.doi.org/10.1215/17358787-3649788

ISSN: $1735-8787$ (electronic)

http://projecteuclid.org/bjma

\title{
CONVEX CONES OF GENERALIZED MULTIPLY MONOTONE FUNCTIONS AND THE DUAL CONES
}

\author{
IOSIF PINELIS
}

Communicated by D. Yang

\begin{abstract}
Let $n$ and $k$ be nonnegative integers such that $1 \leq k \leq n+1$. The convex cone $\mathscr{F}_{+}^{k: n}$ of all functions $f$ on an arbitrary interval $I \subseteq \mathbb{R}$ whose derivatives $f^{(j)}$ of orders $j=k-1, \ldots, n$ are nondecreasing is characterized. A simple description of the convex cone dual to $\mathscr{F}_{+}^{k: n}$ is given. In particular, these results are useful in, and were motivated by, applications in probability. In fact, the results are obtained in a more general setting with certain generalized derivatives of $f$ of the $j$ th order in place of $f^{(j)}$. Somewhat similar results were previously obtained, in terms of Tchebycheff-Markov systems, in the case when the left endpoint of the interval $I$ is finite, with certain additional integrability conditions; such conditions fail to hold in the mentioned applications. Development of substantially new methods was needed to overcome the difficulties.
\end{abstract}

\section{INTRODUCTION}

In applications in probability (see, e.g., [11], [5], [6], [20], [21], [3], [26], [23], [25], and references therein), one is concerned with stochastic domination, defined by a formula of the form

$$
X \succcurlyeq Y \stackrel{\mathscr{F}}{\Longleftrightarrow} X \succcurlyeq Y(\bmod \mathscr{F}) \Longleftrightarrow \mathrm{def} f(X) \geq \mathrm{E} f(Y) \text { for all } f \in \mathscr{F},
$$

Copyright 2016 by the Tusi Mathematical Research Group.

Received Jul. 22, 2015; Accepted Feb. 1, 2016.

2010 Mathematics Subject Classification. Primary 46N10; Secondary 26A48, 26A51, 26A46, 26D05, 26D07, 26D10, 26D15, 34L30, 41A10, 46A55, 49K30, 49M29, 52A07, 52A41, 60E15, 90C25, 90C46.

Keywords. dual cones, multiply monotone functions, generalized moments, stochastic orders, probability inequalities. 


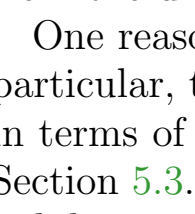

ANALYSIS
Banach J. Math. Anal. 10 (2016), no. 4, 864-897

http://dx.doi.org/10.1215/17358787-3649788

ISSN: $1735-8787$ (electronic)

http://projecteuclid.org/bjma

\title{
CONVEX CONES OF GENERALIZED MULTIPLY MONOTONE FUNCTIONS AND THE DUAL CONES
}

\author{
IOSIF PINELIS
}

Communicated by D. Yang

\begin{abstract}
Let $n$ and $k$ be nonnegative integers such that $1 \leq k \leq n+1$. The convex cone $\mathscr{F}_{+}^{k: n}$ of all functions $f$ on an arbitrary interval $I \subseteq \mathbb{R}$ whose derivatives $f^{(j)}$ of orders $j=k-1, \ldots, n$ are nondecreasing is characterized. A simple description of the convex cone dual to $\mathscr{F}_{+}^{k: n}$ is given. In particular, these results are useful in, and were motivated by, applications in probability. In fact, the results are obtained in a more general setting with certain generalized derivatives of $f$ of the $j$ th order in place of $f^{(j)}$. Somewhat similar results were previously obtained, in terms of Tchebycheff-Markov systems, in the case when the left endpoint of the interval $I$ is finite, with certain additional integrability conditions; such conditions fail to hold in the mentioned applications. Development of substantially new methods was needed to overcome the difficulties.
\end{abstract}

\section{INTRODUCTION}

In applications in probability (see, e.g., [11], [5], [6], [20], [21], [3], [26], [23], [25], and references therein), one is concerned with stochastic domination, defined by a formula of the form

$$
X \succcurlyeq Y \stackrel{\mathscr{F}}{\Longleftrightarrow} X \succcurlyeq Y(\bmod \mathscr{F}) \Longleftrightarrow \mathrm{def} f(X) \geq \mathrm{E} f(Y) \text { for all } f \in \mathscr{F},
$$

Copyright 2016 by the Tusi Mathematical Research Group.

Received Jul. 22, 2015; Accepted Feb. 1, 2016.

2010 Mathematics Subject Classification. Primary 46N10; Secondary 26A48, 26A51, 26A46, 26D05, 26D07, 26D10, 26D15, 34L30, 41A10, 46A55, 49K30, 49M29, 52A07, 52A41, 60E15, 90C25, 90C46.

Keywords. dual cones, multiply monotone functions, generalized moments, stochastic orders, probability inequalities. 


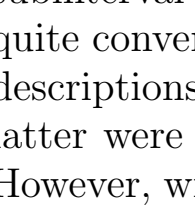

ANALYSIS
Banach J. Math. Anal. 10 (2016), no. 4, 864-897

http://dx.doi.org/10.1215/17358787-3649788

ISSN: $1735-8787$ (electronic)

http://projecteuclid.org/bjma

\title{
CONVEX CONES OF GENERALIZED MULTIPLY MONOTONE FUNCTIONS AND THE DUAL CONES
}

\author{
IOSIF PINELIS
}

Communicated by D. Yang

\begin{abstract}
Let $n$ and $k$ be nonnegative integers such that $1 \leq k \leq n+1$. The convex cone $\mathscr{F}_{+}^{k: n}$ of all functions $f$ on an arbitrary interval $I \subseteq \mathbb{R}$ whose derivatives $f^{(j)}$ of orders $j=k-1, \ldots, n$ are nondecreasing is characterized. A simple description of the convex cone dual to $\mathscr{F}_{+}^{k: n}$ is given. In particular, these results are useful in, and were motivated by, applications in probability. In fact, the results are obtained in a more general setting with certain generalized derivatives of $f$ of the $j$ th order in place of $f^{(j)}$. Somewhat similar results were previously obtained, in terms of Tchebycheff-Markov systems, in the case when the left endpoint of the interval $I$ is finite, with certain additional integrability conditions; such conditions fail to hold in the mentioned applications. Development of substantially new methods was needed to overcome the difficulties.
\end{abstract}

\section{INTRODUCTION}

In applications in probability (see, e.g., [11], [5], [6], [20], [21], [3], [26], [23], [25], and references therein), one is concerned with stochastic domination, defined by a formula of the form

$$
X \succcurlyeq Y \stackrel{\mathscr{F}}{\Longleftrightarrow} X \succcurlyeq Y(\bmod \mathscr{F}) \Longleftrightarrow \mathrm{def} f(X) \geq \mathrm{E} f(Y) \text { for all } f \in \mathscr{F},
$$

Copyright 2016 by the Tusi Mathematical Research Group.

Received Jul. 22, 2015; Accepted Feb. 1, 2016.

2010 Mathematics Subject Classification. Primary 46N10; Secondary 26A48, 26A51, 26A46, 26D05, 26D07, 26D10, 26D15, 34L30, 41A10, 46A55, 49K30, 49M29, 52A07, 52A41, 60E15, 90C25, 90C46.

Keywords. dual cones, multiply monotone functions, generalized moments, stochastic orders, probability inequalities. 


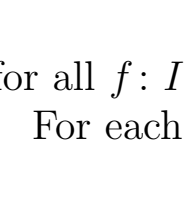

ANALYSIS
Banach J. Math. Anal. 10 (2016), no. 4, 864-897

http://dx.doi.org/10.1215/17358787-3649788

ISSN: $1735-8787$ (electronic)

http://projecteuclid.org/bjma

\title{
CONVEX CONES OF GENERALIZED MULTIPLY MONOTONE FUNCTIONS AND THE DUAL CONES
}

\author{
IOSIF PINELIS
}

Communicated by D. Yang

\begin{abstract}
Let $n$ and $k$ be nonnegative integers such that $1 \leq k \leq n+1$. The convex cone $\mathscr{F}_{+}^{k: n}$ of all functions $f$ on an arbitrary interval $I \subseteq \mathbb{R}$ whose derivatives $f^{(j)}$ of orders $j=k-1, \ldots, n$ are nondecreasing is characterized. A simple description of the convex cone dual to $\mathscr{F}_{+}^{k: n}$ is given. In particular, these results are useful in, and were motivated by, applications in probability. In fact, the results are obtained in a more general setting with certain generalized derivatives of $f$ of the $j$ th order in place of $f^{(j)}$. Somewhat similar results were previously obtained, in terms of Tchebycheff-Markov systems, in the case when the left endpoint of the interval $I$ is finite, with certain additional integrability conditions; such conditions fail to hold in the mentioned applications. Development of substantially new methods was needed to overcome the difficulties.
\end{abstract}

\section{INTRODUCTION}

In applications in probability (see, e.g., [11], [5], [6], [20], [21], [3], [26], [23], [25], and references therein), one is concerned with stochastic domination, defined by a formula of the form

$$
X \succcurlyeq Y \stackrel{\mathscr{F}}{\Longleftrightarrow} X \succcurlyeq Y(\bmod \mathscr{F}) \Longleftrightarrow \mathrm{def} f(X) \geq \mathrm{E} f(Y) \text { for all } f \in \mathscr{F},
$$

Copyright 2016 by the Tusi Mathematical Research Group.

Received Jul. 22, 2015; Accepted Feb. 1, 2016.

2010 Mathematics Subject Classification. Primary 46N10; Secondary 26A48, 26A51, 26A46, 26D05, 26D07, 26D10, 26D15, 34L30, 41A10, 46A55, 49K30, 49M29, 52A07, 52A41, 60E15, 90C25, 90C46.

Keywords. dual cones, multiply monotone functions, generalized moments, stochastic orders, probability inequalities. 


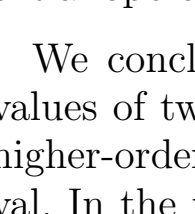

ANALYSIS
Banach J. Math. Anal. 10 (2016), no. 4, 864-897

http://dx.doi.org/10.1215/17358787-3649788

ISSN: $1735-8787$ (electronic)

http://projecteuclid.org/bjma

\title{
CONVEX CONES OF GENERALIZED MULTIPLY MONOTONE FUNCTIONS AND THE DUAL CONES
}

\author{
IOSIF PINELIS
}

Communicated by D. Yang

\begin{abstract}
Let $n$ and $k$ be nonnegative integers such that $1 \leq k \leq n+1$. The convex cone $\mathscr{F}_{+}^{k: n}$ of all functions $f$ on an arbitrary interval $I \subseteq \mathbb{R}$ whose derivatives $f^{(j)}$ of orders $j=k-1, \ldots, n$ are nondecreasing is characterized. A simple description of the convex cone dual to $\mathscr{F}_{+}^{k: n}$ is given. In particular, these results are useful in, and were motivated by, applications in probability. In fact, the results are obtained in a more general setting with certain generalized derivatives of $f$ of the $j$ th order in place of $f^{(j)}$. Somewhat similar results were previously obtained, in terms of Tchebycheff-Markov systems, in the case when the left endpoint of the interval $I$ is finite, with certain additional integrability conditions; such conditions fail to hold in the mentioned applications. Development of substantially new methods was needed to overcome the difficulties.
\end{abstract}

\section{INTRODUCTION}

In applications in probability (see, e.g., [11], [5], [6], [20], [21], [3], [26], [23], [25], and references therein), one is concerned with stochastic domination, defined by a formula of the form

$$
X \succcurlyeq Y \stackrel{\mathscr{F}}{\Longleftrightarrow} X \succcurlyeq Y(\bmod \mathscr{F}) \Longleftrightarrow \mathrm{def} f(X) \geq \mathrm{E} f(Y) \text { for all } f \in \mathscr{F},
$$

Copyright 2016 by the Tusi Mathematical Research Group.

Received Jul. 22, 2015; Accepted Feb. 1, 2016.

2010 Mathematics Subject Classification. Primary 46N10; Secondary 26A48, 26A51, 26A46, 26D05, 26D07, 26D10, 26D15, 34L30, 41A10, 46A55, 49K30, 49M29, 52A07, 52A41, 60E15, 90C25, 90C46.

Keywords. dual cones, multiply monotone functions, generalized moments, stochastic orders, probability inequalities. 


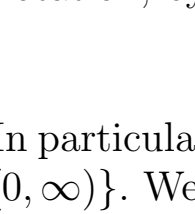

ANALYSIS
Banach J. Math. Anal. 10 (2016), no. 4, 864-897

http://dx.doi.org/10.1215/17358787-3649788

ISSN: $1735-8787$ (electronic)

http://projecteuclid.org/bjma

\title{
CONVEX CONES OF GENERALIZED MULTIPLY MONOTONE FUNCTIONS AND THE DUAL CONES
}

\author{
IOSIF PINELIS
}

Communicated by D. Yang

\begin{abstract}
Let $n$ and $k$ be nonnegative integers such that $1 \leq k \leq n+1$. The convex cone $\mathscr{F}_{+}^{k: n}$ of all functions $f$ on an arbitrary interval $I \subseteq \mathbb{R}$ whose derivatives $f^{(j)}$ of orders $j=k-1, \ldots, n$ are nondecreasing is characterized. A simple description of the convex cone dual to $\mathscr{F}_{+}^{k: n}$ is given. In particular, these results are useful in, and were motivated by, applications in probability. In fact, the results are obtained in a more general setting with certain generalized derivatives of $f$ of the $j$ th order in place of $f^{(j)}$. Somewhat similar results were previously obtained, in terms of Tchebycheff-Markov systems, in the case when the left endpoint of the interval $I$ is finite, with certain additional integrability conditions; such conditions fail to hold in the mentioned applications. Development of substantially new methods was needed to overcome the difficulties.
\end{abstract}

\section{INTRODUCTION}

In applications in probability (see, e.g., [11], [5], [6], [20], [21], [3], [26], [23], [25], and references therein), one is concerned with stochastic domination, defined by a formula of the form

$$
X \succcurlyeq Y \stackrel{\mathscr{F}}{\Longleftrightarrow} X \succcurlyeq Y(\bmod \mathscr{F}) \Longleftrightarrow \mathrm{def} f(X) \geq \mathrm{E} f(Y) \text { for all } f \in \mathscr{F},
$$

Copyright 2016 by the Tusi Mathematical Research Group.

Received Jul. 22, 2015; Accepted Feb. 1, 2016.

2010 Mathematics Subject Classification. Primary 46N10; Secondary 26A48, 26A51, 26A46, 26D05, 26D07, 26D10, 26D15, 34L30, 41A10, 46A55, 49K30, 49M29, 52A07, 52A41, 60E15, 90C25, 90C46.

Keywords. dual cones, multiply monotone functions, generalized moments, stochastic orders, probability inequalities. 


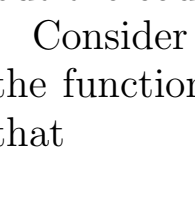

ANALYSIS
Banach J. Math. Anal. 10 (2016), no. 4, 864-897

http://dx.doi.org/10.1215/17358787-3649788

ISSN: $1735-8787$ (electronic)

http://projecteuclid.org/bjma

\title{
CONVEX CONES OF GENERALIZED MULTIPLY MONOTONE FUNCTIONS AND THE DUAL CONES
}

\author{
IOSIF PINELIS
}

Communicated by D. Yang

\begin{abstract}
Let $n$ and $k$ be nonnegative integers such that $1 \leq k \leq n+1$. The convex cone $\mathscr{F}_{+}^{k: n}$ of all functions $f$ on an arbitrary interval $I \subseteq \mathbb{R}$ whose derivatives $f^{(j)}$ of orders $j=k-1, \ldots, n$ are nondecreasing is characterized. A simple description of the convex cone dual to $\mathscr{F}_{+}^{k: n}$ is given. In particular, these results are useful in, and were motivated by, applications in probability. In fact, the results are obtained in a more general setting with certain generalized derivatives of $f$ of the $j$ th order in place of $f^{(j)}$. Somewhat similar results were previously obtained, in terms of Tchebycheff-Markov systems, in the case when the left endpoint of the interval $I$ is finite, with certain additional integrability conditions; such conditions fail to hold in the mentioned applications. Development of substantially new methods was needed to overcome the difficulties.
\end{abstract}

\section{INTRODUCTION}

In applications in probability (see, e.g., [11], [5], [6], [20], [21], [3], [26], [23], [25], and references therein), one is concerned with stochastic domination, defined by a formula of the form

$$
X \succcurlyeq Y \stackrel{\mathscr{F}}{\Longleftrightarrow} X \succcurlyeq Y(\bmod \mathscr{F}) \Longleftrightarrow \mathrm{def} f(X) \geq \mathrm{E} f(Y) \text { for all } f \in \mathscr{F},
$$

Copyright 2016 by the Tusi Mathematical Research Group.

Received Jul. 22, 2015; Accepted Feb. 1, 2016.

2010 Mathematics Subject Classification. Primary 46N10; Secondary 26A48, 26A51, 26A46, 26D05, 26D07, 26D10, 26D15, 34L30, 41A10, 46A55, 49K30, 49M29, 52A07, 52A41, 60E15, 90C25, 90C46.

Keywords. dual cones, multiply monotone functions, generalized moments, stochastic orders, probability inequalities. 


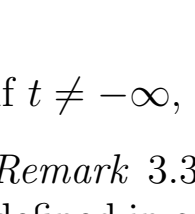

ANALYSIS
Banach J. Math. Anal. 10 (2016), no. 4, 864-897

http://dx.doi.org/10.1215/17358787-3649788

ISSN: $1735-8787$ (electronic)

http://projecteuclid.org/bjma

\title{
CONVEX CONES OF GENERALIZED MULTIPLY MONOTONE FUNCTIONS AND THE DUAL CONES
}

\author{
IOSIF PINELIS
}

Communicated by D. Yang

\begin{abstract}
Let $n$ and $k$ be nonnegative integers such that $1 \leq k \leq n+1$. The convex cone $\mathscr{F}_{+}^{k: n}$ of all functions $f$ on an arbitrary interval $I \subseteq \mathbb{R}$ whose derivatives $f^{(j)}$ of orders $j=k-1, \ldots, n$ are nondecreasing is characterized. A simple description of the convex cone dual to $\mathscr{F}_{+}^{k: n}$ is given. In particular, these results are useful in, and were motivated by, applications in probability. In fact, the results are obtained in a more general setting with certain generalized derivatives of $f$ of the $j$ th order in place of $f^{(j)}$. Somewhat similar results were previously obtained, in terms of Tchebycheff-Markov systems, in the case when the left endpoint of the interval $I$ is finite, with certain additional integrability conditions; such conditions fail to hold in the mentioned applications. Development of substantially new methods was needed to overcome the difficulties.
\end{abstract}

\section{INTRODUCTION}

In applications in probability (see, e.g., [11], [5], [6], [20], [21], [3], [26], [23], [25], and references therein), one is concerned with stochastic domination, defined by a formula of the form

$$
X \succcurlyeq Y \stackrel{\mathscr{F}}{\Longleftrightarrow} X \succcurlyeq Y(\bmod \mathscr{F}) \Longleftrightarrow \mathrm{def} f(X) \geq \mathrm{E} f(Y) \text { for all } f \in \mathscr{F},
$$

Copyright 2016 by the Tusi Mathematical Research Group.

Received Jul. 22, 2015; Accepted Feb. 1, 2016.

2010 Mathematics Subject Classification. Primary 46N10; Secondary 26A48, 26A51, 26A46, 26D05, 26D07, 26D10, 26D15, 34L30, 41A10, 46A55, 49K30, 49M29, 52A07, 52A41, 60E15, 90C25, 90C46.

Keywords. dual cones, multiply monotone functions, generalized moments, stochastic orders, probability inequalities. 


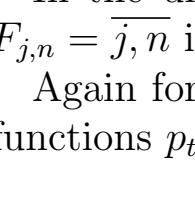

ANALYSIS
Banach J. Math. Anal. 10 (2016), no. 4, 864-897

http://dx.doi.org/10.1215/17358787-3649788

ISSN: $1735-8787$ (electronic)

http://projecteuclid.org/bjma

\title{
CONVEX CONES OF GENERALIZED MULTIPLY MONOTONE FUNCTIONS AND THE DUAL CONES
}

\author{
IOSIF PINELIS
}

Communicated by D. Yang

\begin{abstract}
Let $n$ and $k$ be nonnegative integers such that $1 \leq k \leq n+1$. The convex cone $\mathscr{F}_{+}^{k: n}$ of all functions $f$ on an arbitrary interval $I \subseteq \mathbb{R}$ whose derivatives $f^{(j)}$ of orders $j=k-1, \ldots, n$ are nondecreasing is characterized. A simple description of the convex cone dual to $\mathscr{F}_{+}^{k: n}$ is given. In particular, these results are useful in, and were motivated by, applications in probability. In fact, the results are obtained in a more general setting with certain generalized derivatives of $f$ of the $j$ th order in place of $f^{(j)}$. Somewhat similar results were previously obtained, in terms of Tchebycheff-Markov systems, in the case when the left endpoint of the interval $I$ is finite, with certain additional integrability conditions; such conditions fail to hold in the mentioned applications. Development of substantially new methods was needed to overcome the difficulties.
\end{abstract}

\section{INTRODUCTION}

In applications in probability (see, e.g., [11], [5], [6], [20], [21], [3], [26], [23], [25], and references therein), one is concerned with stochastic domination, defined by a formula of the form

$$
X \succcurlyeq Y \stackrel{\mathscr{F}}{\Longleftrightarrow} X \succcurlyeq Y(\bmod \mathscr{F}) \Longleftrightarrow \mathrm{def} f(X) \geq \mathrm{E} f(Y) \text { for all } f \in \mathscr{F},
$$

Copyright 2016 by the Tusi Mathematical Research Group.

Received Jul. 22, 2015; Accepted Feb. 1, 2016.

2010 Mathematics Subject Classification. Primary 46N10; Secondary 26A48, 26A51, 26A46, 26D05, 26D07, 26D10, 26D15, 34L30, 41A10, 46A55, 49K30, 49M29, 52A07, 52A41, 60E15, 90C25, 90C46.

Keywords. dual cones, multiply monotone functions, generalized moments, stochastic orders, probability inequalities. 


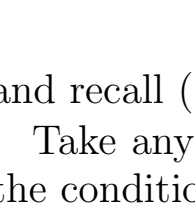

ANALYSIS
Banach J. Math. Anal. 10 (2016), no. 4, 864-897

http://dx.doi.org/10.1215/17358787-3649788

ISSN: $1735-8787$ (electronic)

http://projecteuclid.org/bjma

\title{
CONVEX CONES OF GENERALIZED MULTIPLY MONOTONE FUNCTIONS AND THE DUAL CONES
}

\author{
IOSIF PINELIS
}

Communicated by D. Yang

\begin{abstract}
Let $n$ and $k$ be nonnegative integers such that $1 \leq k \leq n+1$. The convex cone $\mathscr{F}_{+}^{k: n}$ of all functions $f$ on an arbitrary interval $I \subseteq \mathbb{R}$ whose derivatives $f^{(j)}$ of orders $j=k-1, \ldots, n$ are nondecreasing is characterized. A simple description of the convex cone dual to $\mathscr{F}_{+}^{k: n}$ is given. In particular, these results are useful in, and were motivated by, applications in probability. In fact, the results are obtained in a more general setting with certain generalized derivatives of $f$ of the $j$ th order in place of $f^{(j)}$. Somewhat similar results were previously obtained, in terms of Tchebycheff-Markov systems, in the case when the left endpoint of the interval $I$ is finite, with certain additional integrability conditions; such conditions fail to hold in the mentioned applications. Development of substantially new methods was needed to overcome the difficulties.
\end{abstract}

\section{INTRODUCTION}

In applications in probability (see, e.g., [11], [5], [6], [20], [21], [3], [26], [23], [25], and references therein), one is concerned with stochastic domination, defined by a formula of the form

$$
X \succcurlyeq Y \stackrel{\mathscr{F}}{\Longleftrightarrow} X \succcurlyeq Y(\bmod \mathscr{F}) \Longleftrightarrow \mathrm{def} f(X) \geq \mathrm{E} f(Y) \text { for all } f \in \mathscr{F},
$$

Copyright 2016 by the Tusi Mathematical Research Group.

Received Jul. 22, 2015; Accepted Feb. 1, 2016.

2010 Mathematics Subject Classification. Primary 46N10; Secondary 26A48, 26A51, 26A46, 26D05, 26D07, 26D10, 26D15, 34L30, 41A10, 46A55, 49K30, 49M29, 52A07, 52A41, 60E15, 90C25, 90C46.

Keywords. dual cones, multiply monotone functions, generalized moments, stochastic orders, probability inequalities. 


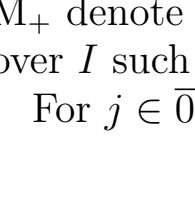

ANALYSIS
Banach J. Math. Anal. 10 (2016), no. 4, 864-897

http://dx.doi.org/10.1215/17358787-3649788

ISSN: $1735-8787$ (electronic)

http://projecteuclid.org/bjma

\title{
CONVEX CONES OF GENERALIZED MULTIPLY MONOTONE FUNCTIONS AND THE DUAL CONES
}

\author{
IOSIF PINELIS
}

Communicated by D. Yang

\begin{abstract}
Let $n$ and $k$ be nonnegative integers such that $1 \leq k \leq n+1$. The convex cone $\mathscr{F}_{+}^{k: n}$ of all functions $f$ on an arbitrary interval $I \subseteq \mathbb{R}$ whose derivatives $f^{(j)}$ of orders $j=k-1, \ldots, n$ are nondecreasing is characterized. A simple description of the convex cone dual to $\mathscr{F}_{+}^{k: n}$ is given. In particular, these results are useful in, and were motivated by, applications in probability. In fact, the results are obtained in a more general setting with certain generalized derivatives of $f$ of the $j$ th order in place of $f^{(j)}$. Somewhat similar results were previously obtained, in terms of Tchebycheff-Markov systems, in the case when the left endpoint of the interval $I$ is finite, with certain additional integrability conditions; such conditions fail to hold in the mentioned applications. Development of substantially new methods was needed to overcome the difficulties.
\end{abstract}

\section{INTRODUCTION}

In applications in probability (see, e.g., [11], [5], [6], [20], [21], [3], [26], [23], [25], and references therein), one is concerned with stochastic domination, defined by a formula of the form

$$
X \succcurlyeq Y \stackrel{\mathscr{F}}{\Longleftrightarrow} X \succcurlyeq Y(\bmod \mathscr{F}) \Longleftrightarrow \mathrm{def} f(X) \geq \mathrm{E} f(Y) \text { for all } f \in \mathscr{F},
$$

Copyright 2016 by the Tusi Mathematical Research Group.

Received Jul. 22, 2015; Accepted Feb. 1, 2016.

2010 Mathematics Subject Classification. Primary 46N10; Secondary 26A48, 26A51, 26A46, 26D05, 26D07, 26D10, 26D15, 34L30, 41A10, 46A55, 49K30, 49M29, 52A07, 52A41, 60E15, 90C25, 90C46.

Keywords. dual cones, multiply monotone functions, generalized moments, stochastic orders, probability inequalities. 


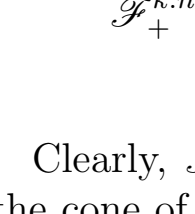

ANALYSIS
Banach J. Math. Anal. 10 (2016), no. 4, 864-897

http://dx.doi.org/10.1215/17358787-3649788

ISSN: $1735-8787$ (electronic)

http://projecteuclid.org/bjma

\title{
CONVEX CONES OF GENERALIZED MULTIPLY MONOTONE FUNCTIONS AND THE DUAL CONES
}

\author{
IOSIF PINELIS
}

Communicated by D. Yang

\begin{abstract}
Let $n$ and $k$ be nonnegative integers such that $1 \leq k \leq n+1$. The convex cone $\mathscr{F}_{+}^{k: n}$ of all functions $f$ on an arbitrary interval $I \subseteq \mathbb{R}$ whose derivatives $f^{(j)}$ of orders $j=k-1, \ldots, n$ are nondecreasing is characterized. A simple description of the convex cone dual to $\mathscr{F}_{+}^{k: n}$ is given. In particular, these results are useful in, and were motivated by, applications in probability. In fact, the results are obtained in a more general setting with certain generalized derivatives of $f$ of the $j$ th order in place of $f^{(j)}$. Somewhat similar results were previously obtained, in terms of Tchebycheff-Markov systems, in the case when the left endpoint of the interval $I$ is finite, with certain additional integrability conditions; such conditions fail to hold in the mentioned applications. Development of substantially new methods was needed to overcome the difficulties.
\end{abstract}

\section{INTRODUCTION}

In applications in probability (see, e.g., [11], [5], [6], [20], [21], [3], [26], [23], [25], and references therein), one is concerned with stochastic domination, defined by a formula of the form

$$
X \succcurlyeq Y \stackrel{\mathscr{F}}{\Longleftrightarrow} X \succcurlyeq Y(\bmod \mathscr{F}) \Longleftrightarrow \mathrm{def} f(X) \geq \mathrm{E} f(Y) \text { for all } f \in \mathscr{F},
$$

Copyright 2016 by the Tusi Mathematical Research Group.

Received Jul. 22, 2015; Accepted Feb. 1, 2016.

2010 Mathematics Subject Classification. Primary 46N10; Secondary 26A48, 26A51, 26A46, 26D05, 26D07, 26D10, 26D15, 34L30, 41A10, 46A55, 49K30, 49M29, 52A07, 52A41, 60E15, 90C25, 90C46.

Keywords. dual cones, multiply monotone functions, generalized moments, stochastic orders, probability inequalities. 


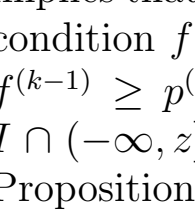

ANALYSIS
Banach J. Math. Anal. 10 (2016), no. 4, 864-897

http://dx.doi.org/10.1215/17358787-3649788

ISSN: $1735-8787$ (electronic)

http://projecteuclid.org/bjma

\title{
CONVEX CONES OF GENERALIZED MULTIPLY MONOTONE FUNCTIONS AND THE DUAL CONES
}

\author{
IOSIF PINELIS
}

Communicated by D. Yang

\begin{abstract}
Let $n$ and $k$ be nonnegative integers such that $1 \leq k \leq n+1$. The convex cone $\mathscr{F}_{+}^{k: n}$ of all functions $f$ on an arbitrary interval $I \subseteq \mathbb{R}$ whose derivatives $f^{(j)}$ of orders $j=k-1, \ldots, n$ are nondecreasing is characterized. A simple description of the convex cone dual to $\mathscr{F}_{+}^{k: n}$ is given. In particular, these results are useful in, and were motivated by, applications in probability. In fact, the results are obtained in a more general setting with certain generalized derivatives of $f$ of the $j$ th order in place of $f^{(j)}$. Somewhat similar results were previously obtained, in terms of Tchebycheff-Markov systems, in the case when the left endpoint of the interval $I$ is finite, with certain additional integrability conditions; such conditions fail to hold in the mentioned applications. Development of substantially new methods was needed to overcome the difficulties.
\end{abstract}

\section{INTRODUCTION}

In applications in probability (see, e.g., [11], [5], [6], [20], [21], [3], [26], [23], [25], and references therein), one is concerned with stochastic domination, defined by a formula of the form

$$
X \succcurlyeq Y \stackrel{\mathscr{F}}{\Longleftrightarrow} X \succcurlyeq Y(\bmod \mathscr{F}) \Longleftrightarrow \mathrm{def} f(X) \geq \mathrm{E} f(Y) \text { for all } f \in \mathscr{F},
$$

Copyright 2016 by the Tusi Mathematical Research Group.

Received Jul. 22, 2015; Accepted Feb. 1, 2016.

2010 Mathematics Subject Classification. Primary 46N10; Secondary 26A48, 26A51, 26A46, 26D05, 26D07, 26D10, 26D15, 34L30, 41A10, 46A55, 49K30, 49M29, 52A07, 52A41, 60E15, 90C25, 90C46.

Keywords. dual cones, multiply monotone functions, generalized moments, stochastic orders, probability inequalities. 


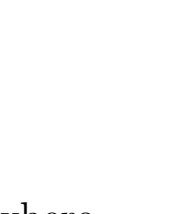

ANALYSIS
Banach J. Math. Anal. 10 (2016), no. 4, 864-897

http://dx.doi.org/10.1215/17358787-3649788

ISSN: $1735-8787$ (electronic)

http://projecteuclid.org/bjma

\title{
CONVEX CONES OF GENERALIZED MULTIPLY MONOTONE FUNCTIONS AND THE DUAL CONES
}

\author{
IOSIF PINELIS
}

Communicated by D. Yang

\begin{abstract}
Let $n$ and $k$ be nonnegative integers such that $1 \leq k \leq n+1$. The convex cone $\mathscr{F}_{+}^{k: n}$ of all functions $f$ on an arbitrary interval $I \subseteq \mathbb{R}$ whose derivatives $f^{(j)}$ of orders $j=k-1, \ldots, n$ are nondecreasing is characterized. A simple description of the convex cone dual to $\mathscr{F}_{+}^{k: n}$ is given. In particular, these results are useful in, and were motivated by, applications in probability. In fact, the results are obtained in a more general setting with certain generalized derivatives of $f$ of the $j$ th order in place of $f^{(j)}$. Somewhat similar results were previously obtained, in terms of Tchebycheff-Markov systems, in the case when the left endpoint of the interval $I$ is finite, with certain additional integrability conditions; such conditions fail to hold in the mentioned applications. Development of substantially new methods was needed to overcome the difficulties.
\end{abstract}

\section{INTRODUCTION}

In applications in probability (see, e.g., [11], [5], [6], [20], [21], [3], [26], [23], [25], and references therein), one is concerned with stochastic domination, defined by a formula of the form

$$
X \succcurlyeq Y \stackrel{\mathscr{F}}{\Longleftrightarrow} X \succcurlyeq Y(\bmod \mathscr{F}) \Longleftrightarrow \mathrm{def} f(X) \geq \mathrm{E} f(Y) \text { for all } f \in \mathscr{F},
$$

Copyright 2016 by the Tusi Mathematical Research Group.

Received Jul. 22, 2015; Accepted Feb. 1, 2016.

2010 Mathematics Subject Classification. Primary 46N10; Secondary 26A48, 26A51, 26A46, 26D05, 26D07, 26D10, 26D15, 34L30, 41A10, 46A55, 49K30, 49M29, 52A07, 52A41, 60E15, 90C25, 90C46.

Keywords. dual cones, multiply monotone functions, generalized moments, stochastic orders, probability inequalities. 


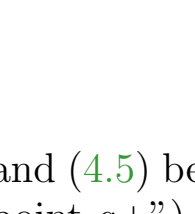

ANALYSIS
Banach J. Math. Anal. 10 (2016), no. 4, 864-897

http://dx.doi.org/10.1215/17358787-3649788

ISSN: $1735-8787$ (electronic)

http://projecteuclid.org/bjma

\title{
CONVEX CONES OF GENERALIZED MULTIPLY MONOTONE FUNCTIONS AND THE DUAL CONES
}

\author{
IOSIF PINELIS
}

Communicated by D. Yang

\begin{abstract}
Let $n$ and $k$ be nonnegative integers such that $1 \leq k \leq n+1$. The convex cone $\mathscr{F}_{+}^{k: n}$ of all functions $f$ on an arbitrary interval $I \subseteq \mathbb{R}$ whose derivatives $f^{(j)}$ of orders $j=k-1, \ldots, n$ are nondecreasing is characterized. A simple description of the convex cone dual to $\mathscr{F}_{+}^{k: n}$ is given. In particular, these results are useful in, and were motivated by, applications in probability. In fact, the results are obtained in a more general setting with certain generalized derivatives of $f$ of the $j$ th order in place of $f^{(j)}$. Somewhat similar results were previously obtained, in terms of Tchebycheff-Markov systems, in the case when the left endpoint of the interval $I$ is finite, with certain additional integrability conditions; such conditions fail to hold in the mentioned applications. Development of substantially new methods was needed to overcome the difficulties.
\end{abstract}

\section{INTRODUCTION}

In applications in probability (see, e.g., [11], [5], [6], [20], [21], [3], [26], [23], [25], and references therein), one is concerned with stochastic domination, defined by a formula of the form

$$
X \succcurlyeq Y \stackrel{\mathscr{F}}{\Longleftrightarrow} X \succcurlyeq Y(\bmod \mathscr{F}) \Longleftrightarrow \mathrm{def} f(X) \geq \mathrm{E} f(Y) \text { for all } f \in \mathscr{F},
$$

Copyright 2016 by the Tusi Mathematical Research Group.

Received Jul. 22, 2015; Accepted Feb. 1, 2016.

2010 Mathematics Subject Classification. Primary 46N10; Secondary 26A48, 26A51, 26A46, 26D05, 26D07, 26D10, 26D15, 34L30, 41A10, 46A55, 49K30, 49M29, 52A07, 52A41, 60E15, 90C25, 90C46.

Keywords. dual cones, multiply monotone functions, generalized moments, stochastic orders, probability inequalities. 


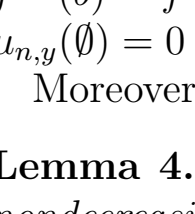

ANALYSIS
Banach J. Math. Anal. 10 (2016), no. 4, 864-897

http://dx.doi.org/10.1215/17358787-3649788

ISSN: $1735-8787$ (electronic)

http://projecteuclid.org/bjma

\title{
CONVEX CONES OF GENERALIZED MULTIPLY MONOTONE FUNCTIONS AND THE DUAL CONES
}

\author{
IOSIF PINELIS
}

Communicated by D. Yang

\begin{abstract}
Let $n$ and $k$ be nonnegative integers such that $1 \leq k \leq n+1$. The convex cone $\mathscr{F}_{+}^{k: n}$ of all functions $f$ on an arbitrary interval $I \subseteq \mathbb{R}$ whose derivatives $f^{(j)}$ of orders $j=k-1, \ldots, n$ are nondecreasing is characterized. A simple description of the convex cone dual to $\mathscr{F}_{+}^{k: n}$ is given. In particular, these results are useful in, and were motivated by, applications in probability. In fact, the results are obtained in a more general setting with certain generalized derivatives of $f$ of the $j$ th order in place of $f^{(j)}$. Somewhat similar results were previously obtained, in terms of Tchebycheff-Markov systems, in the case when the left endpoint of the interval $I$ is finite, with certain additional integrability conditions; such conditions fail to hold in the mentioned applications. Development of substantially new methods was needed to overcome the difficulties.
\end{abstract}

\section{INTRODUCTION}

In applications in probability (see, e.g., [11], [5], [6], [20], [21], [3], [26], [23], [25], and references therein), one is concerned with stochastic domination, defined by a formula of the form

$$
X \succcurlyeq Y \stackrel{\mathscr{F}}{\Longleftrightarrow} X \succcurlyeq Y(\bmod \mathscr{F}) \Longleftrightarrow \mathrm{def} f(X) \geq \mathrm{E} f(Y) \text { for all } f \in \mathscr{F},
$$

Copyright 2016 by the Tusi Mathematical Research Group.

Received Jul. 22, 2015; Accepted Feb. 1, 2016.

2010 Mathematics Subject Classification. Primary 46N10; Secondary 26A48, 26A51, 26A46, 26D05, 26D07, 26D10, 26D15, 34L30, 41A10, 46A55, 49K30, 49M29, 52A07, 52A41, 60E15, 90C25, 90C46.

Keywords. dual cones, multiply monotone functions, generalized moments, stochastic orders, probability inequalities. 


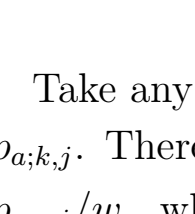

ANALYSIS
Banach J. Math. Anal. 10 (2016), no. 4, 864-897

http://dx.doi.org/10.1215/17358787-3649788

ISSN: $1735-8787$ (electronic)

http://projecteuclid.org/bjma

\title{
CONVEX CONES OF GENERALIZED MULTIPLY MONOTONE FUNCTIONS AND THE DUAL CONES
}

\author{
IOSIF PINELIS
}

Communicated by D. Yang

\begin{abstract}
Let $n$ and $k$ be nonnegative integers such that $1 \leq k \leq n+1$. The convex cone $\mathscr{F}_{+}^{k: n}$ of all functions $f$ on an arbitrary interval $I \subseteq \mathbb{R}$ whose derivatives $f^{(j)}$ of orders $j=k-1, \ldots, n$ are nondecreasing is characterized. A simple description of the convex cone dual to $\mathscr{F}_{+}^{k: n}$ is given. In particular, these results are useful in, and were motivated by, applications in probability. In fact, the results are obtained in a more general setting with certain generalized derivatives of $f$ of the $j$ th order in place of $f^{(j)}$. Somewhat similar results were previously obtained, in terms of Tchebycheff-Markov systems, in the case when the left endpoint of the interval $I$ is finite, with certain additional integrability conditions; such conditions fail to hold in the mentioned applications. Development of substantially new methods was needed to overcome the difficulties.
\end{abstract}

\section{INTRODUCTION}

In applications in probability (see, e.g., [11], [5], [6], [20], [21], [3], [26], [23], [25], and references therein), one is concerned with stochastic domination, defined by a formula of the form

$$
X \succcurlyeq Y \stackrel{\mathscr{F}}{\Longleftrightarrow} X \succcurlyeq Y(\bmod \mathscr{F}) \Longleftrightarrow \mathrm{def} f(X) \geq \mathrm{E} f(Y) \text { for all } f \in \mathscr{F},
$$

Copyright 2016 by the Tusi Mathematical Research Group.

Received Jul. 22, 2015; Accepted Feb. 1, 2016.

2010 Mathematics Subject Classification. Primary 46N10; Secondary 26A48, 26A51, 26A46, 26D05, 26D07, 26D10, 26D15, 34L30, 41A10, 46A55, 49K30, 49M29, 52A07, 52A41, 60E15, 90C25, 90C46.

Keywords. dual cones, multiply monotone functions, generalized moments, stochastic orders, probability inequalities. 


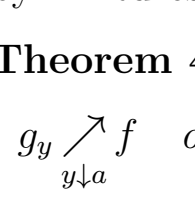

ANALYSIS
Banach J. Math. Anal. 10 (2016), no. 4, 864-897

http://dx.doi.org/10.1215/17358787-3649788

ISSN: $1735-8787$ (electronic)

http://projecteuclid.org/bjma

\title{
CONVEX CONES OF GENERALIZED MULTIPLY MONOTONE FUNCTIONS AND THE DUAL CONES
}

\author{
IOSIF PINELIS
}

Communicated by D. Yang

\begin{abstract}
Let $n$ and $k$ be nonnegative integers such that $1 \leq k \leq n+1$. The convex cone $\mathscr{F}_{+}^{k: n}$ of all functions $f$ on an arbitrary interval $I \subseteq \mathbb{R}$ whose derivatives $f^{(j)}$ of orders $j=k-1, \ldots, n$ are nondecreasing is characterized. A simple description of the convex cone dual to $\mathscr{F}_{+}^{k: n}$ is given. In particular, these results are useful in, and were motivated by, applications in probability. In fact, the results are obtained in a more general setting with certain generalized derivatives of $f$ of the $j$ th order in place of $f^{(j)}$. Somewhat similar results were previously obtained, in terms of Tchebycheff-Markov systems, in the case when the left endpoint of the interval $I$ is finite, with certain additional integrability conditions; such conditions fail to hold in the mentioned applications. Development of substantially new methods was needed to overcome the difficulties.
\end{abstract}

\section{INTRODUCTION}

In applications in probability (see, e.g., [11], [5], [6], [20], [21], [3], [26], [23], [25], and references therein), one is concerned with stochastic domination, defined by a formula of the form

$$
X \succcurlyeq Y \stackrel{\mathscr{F}}{\Longleftrightarrow} X \succcurlyeq Y(\bmod \mathscr{F}) \Longleftrightarrow \mathrm{def} f(X) \geq \mathrm{E} f(Y) \text { for all } f \in \mathscr{F},
$$

Copyright 2016 by the Tusi Mathematical Research Group.

Received Jul. 22, 2015; Accepted Feb. 1, 2016.

2010 Mathematics Subject Classification. Primary 46N10; Secondary 26A48, 26A51, 26A46, 26D05, 26D07, 26D10, 26D15, 34L30, 41A10, 46A55, 49K30, 49M29, 52A07, 52A41, 60E15, 90C25, 90C46.

Keywords. dual cones, multiply monotone functions, generalized moments, stochastic orders, probability inequalities. 


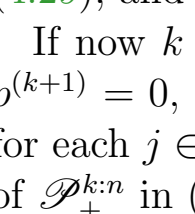

ANALYSIS
Banach J. Math. Anal. 10 (2016), no. 4, 864-897

http://dx.doi.org/10.1215/17358787-3649788

ISSN: $1735-8787$ (electronic)

http://projecteuclid.org/bjma

\title{
CONVEX CONES OF GENERALIZED MULTIPLY MONOTONE FUNCTIONS AND THE DUAL CONES
}

\author{
IOSIF PINELIS
}

Communicated by D. Yang

\begin{abstract}
Let $n$ and $k$ be nonnegative integers such that $1 \leq k \leq n+1$. The convex cone $\mathscr{F}_{+}^{k: n}$ of all functions $f$ on an arbitrary interval $I \subseteq \mathbb{R}$ whose derivatives $f^{(j)}$ of orders $j=k-1, \ldots, n$ are nondecreasing is characterized. A simple description of the convex cone dual to $\mathscr{F}_{+}^{k: n}$ is given. In particular, these results are useful in, and were motivated by, applications in probability. In fact, the results are obtained in a more general setting with certain generalized derivatives of $f$ of the $j$ th order in place of $f^{(j)}$. Somewhat similar results were previously obtained, in terms of Tchebycheff-Markov systems, in the case when the left endpoint of the interval $I$ is finite, with certain additional integrability conditions; such conditions fail to hold in the mentioned applications. Development of substantially new methods was needed to overcome the difficulties.
\end{abstract}

\section{INTRODUCTION}

In applications in probability (see, e.g., [11], [5], [6], [20], [21], [3], [26], [23], [25], and references therein), one is concerned with stochastic domination, defined by a formula of the form

$$
X \succcurlyeq Y \stackrel{\mathscr{F}}{\Longleftrightarrow} X \succcurlyeq Y(\bmod \mathscr{F}) \Longleftrightarrow \mathrm{def} f(X) \geq \mathrm{E} f(Y) \text { for all } f \in \mathscr{F},
$$

Copyright 2016 by the Tusi Mathematical Research Group.

Received Jul. 22, 2015; Accepted Feb. 1, 2016.

2010 Mathematics Subject Classification. Primary 46N10; Secondary 26A48, 26A51, 26A46, 26D05, 26D07, 26D10, 26D15, 34L30, 41A10, 46A55, 49K30, 49M29, 52A07, 52A41, 60E15, 90C25, 90C46.

Keywords. dual cones, multiply monotone functions, generalized moments, stochastic orders, probability inequalities. 


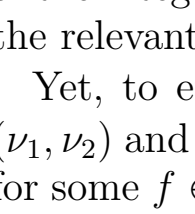

ANALYSIS
Banach J. Math. Anal. 10 (2016), no. 4, 864-897

http://dx.doi.org/10.1215/17358787-3649788

ISSN: $1735-8787$ (electronic)

http://projecteuclid.org/bjma

\title{
CONVEX CONES OF GENERALIZED MULTIPLY MONOTONE FUNCTIONS AND THE DUAL CONES
}

\author{
IOSIF PINELIS
}

Communicated by D. Yang

\begin{abstract}
Let $n$ and $k$ be nonnegative integers such that $1 \leq k \leq n+1$. The convex cone $\mathscr{F}_{+}^{k: n}$ of all functions $f$ on an arbitrary interval $I \subseteq \mathbb{R}$ whose derivatives $f^{(j)}$ of orders $j=k-1, \ldots, n$ are nondecreasing is characterized. A simple description of the convex cone dual to $\mathscr{F}_{+}^{k: n}$ is given. In particular, these results are useful in, and were motivated by, applications in probability. In fact, the results are obtained in a more general setting with certain generalized derivatives of $f$ of the $j$ th order in place of $f^{(j)}$. Somewhat similar results were previously obtained, in terms of Tchebycheff-Markov systems, in the case when the left endpoint of the interval $I$ is finite, with certain additional integrability conditions; such conditions fail to hold in the mentioned applications. Development of substantially new methods was needed to overcome the difficulties.
\end{abstract}

\section{INTRODUCTION}

In applications in probability (see, e.g., [11], [5], [6], [20], [21], [3], [26], [23], [25], and references therein), one is concerned with stochastic domination, defined by a formula of the form

$$
X \succcurlyeq Y \stackrel{\mathscr{F}}{\Longleftrightarrow} X \succcurlyeq Y(\bmod \mathscr{F}) \Longleftrightarrow \mathrm{def} f(X) \geq \mathrm{E} f(Y) \text { for all } f \in \mathscr{F},
$$

Copyright 2016 by the Tusi Mathematical Research Group.

Received Jul. 22, 2015; Accepted Feb. 1, 2016.

2010 Mathematics Subject Classification. Primary 46N10; Secondary 26A48, 26A51, 26A46, 26D05, 26D07, 26D10, 26D15, 34L30, 41A10, 46A55, 49K30, 49M29, 52A07, 52A41, 60E15, 90C25, 90C46.

Keywords. dual cones, multiply monotone functions, generalized moments, stochastic orders, probability inequalities. 


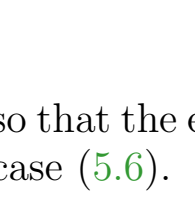

ANALYSIS
Banach J. Math. Anal. 10 (2016), no. 4, 864-897

http://dx.doi.org/10.1215/17358787-3649788

ISSN: $1735-8787$ (electronic)

http://projecteuclid.org/bjma

\title{
CONVEX CONES OF GENERALIZED MULTIPLY MONOTONE FUNCTIONS AND THE DUAL CONES
}

\author{
IOSIF PINELIS
}

Communicated by D. Yang

\begin{abstract}
Let $n$ and $k$ be nonnegative integers such that $1 \leq k \leq n+1$. The convex cone $\mathscr{F}_{+}^{k: n}$ of all functions $f$ on an arbitrary interval $I \subseteq \mathbb{R}$ whose derivatives $f^{(j)}$ of orders $j=k-1, \ldots, n$ are nondecreasing is characterized. A simple description of the convex cone dual to $\mathscr{F}_{+}^{k: n}$ is given. In particular, these results are useful in, and were motivated by, applications in probability. In fact, the results are obtained in a more general setting with certain generalized derivatives of $f$ of the $j$ th order in place of $f^{(j)}$. Somewhat similar results were previously obtained, in terms of Tchebycheff-Markov systems, in the case when the left endpoint of the interval $I$ is finite, with certain additional integrability conditions; such conditions fail to hold in the mentioned applications. Development of substantially new methods was needed to overcome the difficulties.
\end{abstract}

\section{INTRODUCTION}

In applications in probability (see, e.g., [11], [5], [6], [20], [21], [3], [26], [23], [25], and references therein), one is concerned with stochastic domination, defined by a formula of the form

$$
X \succcurlyeq Y \stackrel{\mathscr{F}}{\Longleftrightarrow} X \succcurlyeq Y(\bmod \mathscr{F}) \Longleftrightarrow \mathrm{def} f(X) \geq \mathrm{E} f(Y) \text { for all } f \in \mathscr{F},
$$

Copyright 2016 by the Tusi Mathematical Research Group.

Received Jul. 22, 2015; Accepted Feb. 1, 2016.

2010 Mathematics Subject Classification. Primary 46N10; Secondary 26A48, 26A51, 26A46, 26D05, 26D07, 26D10, 26D15, 34L30, 41A10, 46A55, 49K30, 49M29, 52A07, 52A41, 60E15, 90C25, 90C46.

Keywords. dual cones, multiply monotone functions, generalized moments, stochastic orders, probability inequalities. 


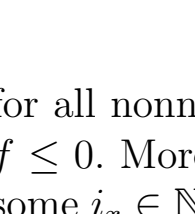

ANALYSIS
Banach J. Math. Anal. 10 (2016), no. 4, 864-897

http://dx.doi.org/10.1215/17358787-3649788

ISSN: $1735-8787$ (electronic)

http://projecteuclid.org/bjma

\title{
CONVEX CONES OF GENERALIZED MULTIPLY MONOTONE FUNCTIONS AND THE DUAL CONES
}

\author{
IOSIF PINELIS
}

Communicated by D. Yang

\begin{abstract}
Let $n$ and $k$ be nonnegative integers such that $1 \leq k \leq n+1$. The convex cone $\mathscr{F}_{+}^{k: n}$ of all functions $f$ on an arbitrary interval $I \subseteq \mathbb{R}$ whose derivatives $f^{(j)}$ of orders $j=k-1, \ldots, n$ are nondecreasing is characterized. A simple description of the convex cone dual to $\mathscr{F}_{+}^{k: n}$ is given. In particular, these results are useful in, and were motivated by, applications in probability. In fact, the results are obtained in a more general setting with certain generalized derivatives of $f$ of the $j$ th order in place of $f^{(j)}$. Somewhat similar results were previously obtained, in terms of Tchebycheff-Markov systems, in the case when the left endpoint of the interval $I$ is finite, with certain additional integrability conditions; such conditions fail to hold in the mentioned applications. Development of substantially new methods was needed to overcome the difficulties.
\end{abstract}

\section{INTRODUCTION}

In applications in probability (see, e.g., [11], [5], [6], [20], [21], [3], [26], [23], [25], and references therein), one is concerned with stochastic domination, defined by a formula of the form

$$
X \succcurlyeq Y \stackrel{\mathscr{F}}{\Longleftrightarrow} X \succcurlyeq Y(\bmod \mathscr{F}) \Longleftrightarrow \mathrm{def} f(X) \geq \mathrm{E} f(Y) \text { for all } f \in \mathscr{F},
$$

Copyright 2016 by the Tusi Mathematical Research Group.

Received Jul. 22, 2015; Accepted Feb. 1, 2016.

2010 Mathematics Subject Classification. Primary 46N10; Secondary 26A48, 26A51, 26A46, 26D05, 26D07, 26D10, 26D15, 34L30, 41A10, 46A55, 49K30, 49M29, 52A07, 52A41, 60E15, 90C25, 90C46.

Keywords. dual cones, multiply monotone functions, generalized moments, stochastic orders, probability inequalities. 


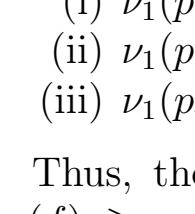

ANALYSIS
Banach J. Math. Anal. 10 (2016), no. 4, 864-897

http://dx.doi.org/10.1215/17358787-3649788

ISSN: $1735-8787$ (electronic)

http://projecteuclid.org/bjma

\title{
CONVEX CONES OF GENERALIZED MULTIPLY MONOTONE FUNCTIONS AND THE DUAL CONES
}

\author{
IOSIF PINELIS
}

Communicated by D. Yang

\begin{abstract}
Let $n$ and $k$ be nonnegative integers such that $1 \leq k \leq n+1$. The convex cone $\mathscr{F}_{+}^{k: n}$ of all functions $f$ on an arbitrary interval $I \subseteq \mathbb{R}$ whose derivatives $f^{(j)}$ of orders $j=k-1, \ldots, n$ are nondecreasing is characterized. A simple description of the convex cone dual to $\mathscr{F}_{+}^{k: n}$ is given. In particular, these results are useful in, and were motivated by, applications in probability. In fact, the results are obtained in a more general setting with certain generalized derivatives of $f$ of the $j$ th order in place of $f^{(j)}$. Somewhat similar results were previously obtained, in terms of Tchebycheff-Markov systems, in the case when the left endpoint of the interval $I$ is finite, with certain additional integrability conditions; such conditions fail to hold in the mentioned applications. Development of substantially new methods was needed to overcome the difficulties.
\end{abstract}

\section{INTRODUCTION}

In applications in probability (see, e.g., [11], [5], [6], [20], [21], [3], [26], [23], [25], and references therein), one is concerned with stochastic domination, defined by a formula of the form

$$
X \succcurlyeq Y \stackrel{\mathscr{F}}{\Longleftrightarrow} X \succcurlyeq Y(\bmod \mathscr{F}) \Longleftrightarrow \mathrm{def} f(X) \geq \mathrm{E} f(Y) \text { for all } f \in \mathscr{F},
$$

Copyright 2016 by the Tusi Mathematical Research Group.

Received Jul. 22, 2015; Accepted Feb. 1, 2016.

2010 Mathematics Subject Classification. Primary 46N10; Secondary 26A48, 26A51, 26A46, 26D05, 26D07, 26D10, 26D15, 34L30, 41A10, 46A55, 49K30, 49M29, 52A07, 52A41, 60E15, 90C25, 90C46.

Keywords. dual cones, multiply monotone functions, generalized moments, stochastic orders, probability inequalities. 


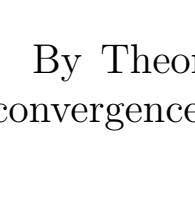

ANALYSIS
Banach J. Math. Anal. 10 (2016), no. 4, 864-897

http://dx.doi.org/10.1215/17358787-3649788

ISSN: $1735-8787$ (electronic)

http://projecteuclid.org/bjma

\title{
CONVEX CONES OF GENERALIZED MULTIPLY MONOTONE FUNCTIONS AND THE DUAL CONES
}

\author{
IOSIF PINELIS
}

Communicated by D. Yang

\begin{abstract}
Let $n$ and $k$ be nonnegative integers such that $1 \leq k \leq n+1$. The convex cone $\mathscr{F}_{+}^{k: n}$ of all functions $f$ on an arbitrary interval $I \subseteq \mathbb{R}$ whose derivatives $f^{(j)}$ of orders $j=k-1, \ldots, n$ are nondecreasing is characterized. A simple description of the convex cone dual to $\mathscr{F}_{+}^{k: n}$ is given. In particular, these results are useful in, and were motivated by, applications in probability. In fact, the results are obtained in a more general setting with certain generalized derivatives of $f$ of the $j$ th order in place of $f^{(j)}$. Somewhat similar results were previously obtained, in terms of Tchebycheff-Markov systems, in the case when the left endpoint of the interval $I$ is finite, with certain additional integrability conditions; such conditions fail to hold in the mentioned applications. Development of substantially new methods was needed to overcome the difficulties.
\end{abstract}

\section{INTRODUCTION}

In applications in probability (see, e.g., [11], [5], [6], [20], [21], [3], [26], [23], [25], and references therein), one is concerned with stochastic domination, defined by a formula of the form

$$
X \succcurlyeq Y \stackrel{\mathscr{F}}{\Longleftrightarrow} X \succcurlyeq Y(\bmod \mathscr{F}) \Longleftrightarrow \mathrm{def} f(X) \geq \mathrm{E} f(Y) \text { for all } f \in \mathscr{F},
$$

Copyright 2016 by the Tusi Mathematical Research Group.

Received Jul. 22, 2015; Accepted Feb. 1, 2016.

2010 Mathematics Subject Classification. Primary 46N10; Secondary 26A48, 26A51, 26A46, 26D05, 26D07, 26D10, 26D15, 34L30, 41A10, 46A55, 49K30, 49M29, 52A07, 52A41, 60E15, 90C25, 90C46.

Keywords. dual cones, multiply monotone functions, generalized moments, stochastic orders, probability inequalities. 


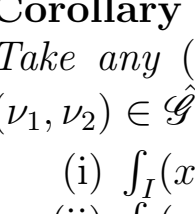

ANALYSIS
Banach J. Math. Anal. 10 (2016), no. 4, 864-897

http://dx.doi.org/10.1215/17358787-3649788

ISSN: $1735-8787$ (electronic)

http://projecteuclid.org/bjma

\title{
CONVEX CONES OF GENERALIZED MULTIPLY MONOTONE FUNCTIONS AND THE DUAL CONES
}

\author{
IOSIF PINELIS
}

Communicated by D. Yang

\begin{abstract}
Let $n$ and $k$ be nonnegative integers such that $1 \leq k \leq n+1$. The convex cone $\mathscr{F}_{+}^{k: n}$ of all functions $f$ on an arbitrary interval $I \subseteq \mathbb{R}$ whose derivatives $f^{(j)}$ of orders $j=k-1, \ldots, n$ are nondecreasing is characterized. A simple description of the convex cone dual to $\mathscr{F}_{+}^{k: n}$ is given. In particular, these results are useful in, and were motivated by, applications in probability. In fact, the results are obtained in a more general setting with certain generalized derivatives of $f$ of the $j$ th order in place of $f^{(j)}$. Somewhat similar results were previously obtained, in terms of Tchebycheff-Markov systems, in the case when the left endpoint of the interval $I$ is finite, with certain additional integrability conditions; such conditions fail to hold in the mentioned applications. Development of substantially new methods was needed to overcome the difficulties.
\end{abstract}

\section{INTRODUCTION}

In applications in probability (see, e.g., [11], [5], [6], [20], [21], [3], [26], [23], [25], and references therein), one is concerned with stochastic domination, defined by a formula of the form

$$
X \succcurlyeq Y \stackrel{\mathscr{F}}{\Longleftrightarrow} X \succcurlyeq Y(\bmod \mathscr{F}) \Longleftrightarrow \mathrm{def} f(X) \geq \mathrm{E} f(Y) \text { for all } f \in \mathscr{F},
$$

Copyright 2016 by the Tusi Mathematical Research Group.

Received Jul. 22, 2015; Accepted Feb. 1, 2016.

2010 Mathematics Subject Classification. Primary 46N10; Secondary 26A48, 26A51, 26A46, 26D05, 26D07, 26D10, 26D15, 34L30, 41A10, 46A55, 49K30, 49M29, 52A07, 52A41, 60E15, 90C25, 90C46.

Keywords. dual cones, multiply monotone functions, generalized moments, stochastic orders, probability inequalities. 


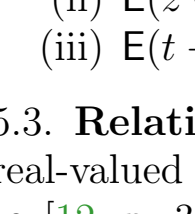

ANALYSIS
Banach J. Math. Anal. 10 (2016), no. 4, 864-897

http://dx.doi.org/10.1215/17358787-3649788

ISSN: $1735-8787$ (electronic)

http://projecteuclid.org/bjma

\title{
CONVEX CONES OF GENERALIZED MULTIPLY MONOTONE FUNCTIONS AND THE DUAL CONES
}

\author{
IOSIF PINELIS
}

Communicated by D. Yang

\begin{abstract}
Let $n$ and $k$ be nonnegative integers such that $1 \leq k \leq n+1$. The convex cone $\mathscr{F}_{+}^{k: n}$ of all functions $f$ on an arbitrary interval $I \subseteq \mathbb{R}$ whose derivatives $f^{(j)}$ of orders $j=k-1, \ldots, n$ are nondecreasing is characterized. A simple description of the convex cone dual to $\mathscr{F}_{+}^{k: n}$ is given. In particular, these results are useful in, and were motivated by, applications in probability. In fact, the results are obtained in a more general setting with certain generalized derivatives of $f$ of the $j$ th order in place of $f^{(j)}$. Somewhat similar results were previously obtained, in terms of Tchebycheff-Markov systems, in the case when the left endpoint of the interval $I$ is finite, with certain additional integrability conditions; such conditions fail to hold in the mentioned applications. Development of substantially new methods was needed to overcome the difficulties.
\end{abstract}

\section{INTRODUCTION}

In applications in probability (see, e.g., [11], [5], [6], [20], [21], [3], [26], [23], [25], and references therein), one is concerned with stochastic domination, defined by a formula of the form

$$
X \succcurlyeq Y \stackrel{\mathscr{F}}{\Longleftrightarrow} X \succcurlyeq Y(\bmod \mathscr{F}) \Longleftrightarrow \mathrm{def} f(X) \geq \mathrm{E} f(Y) \text { for all } f \in \mathscr{F},
$$

Copyright 2016 by the Tusi Mathematical Research Group.

Received Jul. 22, 2015; Accepted Feb. 1, 2016.

2010 Mathematics Subject Classification. Primary 46N10; Secondary 26A48, 26A51, 26A46, 26D05, 26D07, 26D10, 26D15, 34L30, 41A10, 46A55, 49K30, 49M29, 52A07, 52A41, 60E15, 90C25, 90C46.

Keywords. dual cones, multiply monotone functions, generalized moments, stochastic orders, probability inequalities. 


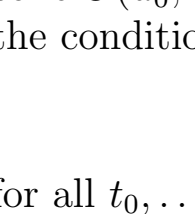

ANALYSIS
Banach J. Math. Anal. 10 (2016), no. 4, 864-897

http://dx.doi.org/10.1215/17358787-3649788

ISSN: $1735-8787$ (electronic)

http://projecteuclid.org/bjma

\title{
CONVEX CONES OF GENERALIZED MULTIPLY MONOTONE FUNCTIONS AND THE DUAL CONES
}

\author{
IOSIF PINELIS
}

Communicated by D. Yang

\begin{abstract}
Let $n$ and $k$ be nonnegative integers such that $1 \leq k \leq n+1$. The convex cone $\mathscr{F}_{+}^{k: n}$ of all functions $f$ on an arbitrary interval $I \subseteq \mathbb{R}$ whose derivatives $f^{(j)}$ of orders $j=k-1, \ldots, n$ are nondecreasing is characterized. A simple description of the convex cone dual to $\mathscr{F}_{+}^{k: n}$ is given. In particular, these results are useful in, and were motivated by, applications in probability. In fact, the results are obtained in a more general setting with certain generalized derivatives of $f$ of the $j$ th order in place of $f^{(j)}$. Somewhat similar results were previously obtained, in terms of Tchebycheff-Markov systems, in the case when the left endpoint of the interval $I$ is finite, with certain additional integrability conditions; such conditions fail to hold in the mentioned applications. Development of substantially new methods was needed to overcome the difficulties.
\end{abstract}

\section{INTRODUCTION}

In applications in probability (see, e.g., [11], [5], [6], [20], [21], [3], [26], [23], [25], and references therein), one is concerned with stochastic domination, defined by a formula of the form

$$
X \succcurlyeq Y \stackrel{\mathscr{F}}{\Longleftrightarrow} X \succcurlyeq Y(\bmod \mathscr{F}) \Longleftrightarrow \mathrm{def} f(X) \geq \mathrm{E} f(Y) \text { for all } f \in \mathscr{F},
$$

Copyright 2016 by the Tusi Mathematical Research Group.

Received Jul. 22, 2015; Accepted Feb. 1, 2016.

2010 Mathematics Subject Classification. Primary 46N10; Secondary 26A48, 26A51, 26A46, 26D05, 26D07, 26D10, 26D15, 34L30, 41A10, 46A55, 49K30, 49M29, 52A07, 52A41, 60E15, 90C25, 90C46.

Keywords. dual cones, multiply monotone functions, generalized moments, stochastic orders, probability inequalities. 


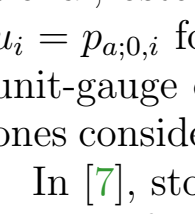

ANALYSIS
Banach J. Math. Anal. 10 (2016), no. 4, 864-897

http://dx.doi.org/10.1215/17358787-3649788

ISSN: $1735-8787$ (electronic)

http://projecteuclid.org/bjma

\title{
CONVEX CONES OF GENERALIZED MULTIPLY MONOTONE FUNCTIONS AND THE DUAL CONES
}

\author{
IOSIF PINELIS
}

Communicated by D. Yang

\begin{abstract}
Let $n$ and $k$ be nonnegative integers such that $1 \leq k \leq n+1$. The convex cone $\mathscr{F}_{+}^{k: n}$ of all functions $f$ on an arbitrary interval $I \subseteq \mathbb{R}$ whose derivatives $f^{(j)}$ of orders $j=k-1, \ldots, n$ are nondecreasing is characterized. A simple description of the convex cone dual to $\mathscr{F}_{+}^{k: n}$ is given. In particular, these results are useful in, and were motivated by, applications in probability. In fact, the results are obtained in a more general setting with certain generalized derivatives of $f$ of the $j$ th order in place of $f^{(j)}$. Somewhat similar results were previously obtained, in terms of Tchebycheff-Markov systems, in the case when the left endpoint of the interval $I$ is finite, with certain additional integrability conditions; such conditions fail to hold in the mentioned applications. Development of substantially new methods was needed to overcome the difficulties.
\end{abstract}

\section{INTRODUCTION}

In applications in probability (see, e.g., [11], [5], [6], [20], [21], [3], [26], [23], [25], and references therein), one is concerned with stochastic domination, defined by a formula of the form

$$
X \succcurlyeq Y \stackrel{\mathscr{F}}{\Longleftrightarrow} X \succcurlyeq Y(\bmod \mathscr{F}) \Longleftrightarrow \mathrm{def} f(X) \geq \mathrm{E} f(Y) \text { for all } f \in \mathscr{F},
$$

Copyright 2016 by the Tusi Mathematical Research Group.

Received Jul. 22, 2015; Accepted Feb. 1, 2016.

2010 Mathematics Subject Classification. Primary 46N10; Secondary 26A48, 26A51, 26A46, 26D05, 26D07, 26D10, 26D15, 34L30, 41A10, 46A55, 49K30, 49M29, 52A07, 52A41, 60E15, 90C25, 90C46.

Keywords. dual cones, multiply monotone functions, generalized moments, stochastic orders, probability inequalities. 


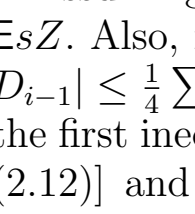

ANALYSIS
Banach J. Math. Anal. 10 (2016), no. 4, 864-897

http://dx.doi.org/10.1215/17358787-3649788

ISSN: $1735-8787$ (electronic)

http://projecteuclid.org/bjma

\title{
CONVEX CONES OF GENERALIZED MULTIPLY MONOTONE FUNCTIONS AND THE DUAL CONES
}

\author{
IOSIF PINELIS
}

Communicated by D. Yang

\begin{abstract}
Let $n$ and $k$ be nonnegative integers such that $1 \leq k \leq n+1$. The convex cone $\mathscr{F}_{+}^{k: n}$ of all functions $f$ on an arbitrary interval $I \subseteq \mathbb{R}$ whose derivatives $f^{(j)}$ of orders $j=k-1, \ldots, n$ are nondecreasing is characterized. A simple description of the convex cone dual to $\mathscr{F}_{+}^{k: n}$ is given. In particular, these results are useful in, and were motivated by, applications in probability. In fact, the results are obtained in a more general setting with certain generalized derivatives of $f$ of the $j$ th order in place of $f^{(j)}$. Somewhat similar results were previously obtained, in terms of Tchebycheff-Markov systems, in the case when the left endpoint of the interval $I$ is finite, with certain additional integrability conditions; such conditions fail to hold in the mentioned applications. Development of substantially new methods was needed to overcome the difficulties.
\end{abstract}

\section{INTRODUCTION}

In applications in probability (see, e.g., [11], [5], [6], [20], [21], [3], [26], [23], [25], and references therein), one is concerned with stochastic domination, defined by a formula of the form

$$
X \succcurlyeq Y \stackrel{\mathscr{F}}{\Longleftrightarrow} X \succcurlyeq Y(\bmod \mathscr{F}) \Longleftrightarrow \mathrm{def} f(X) \geq \mathrm{E} f(Y) \text { for all } f \in \mathscr{F},
$$

Copyright 2016 by the Tusi Mathematical Research Group.

Received Jul. 22, 2015; Accepted Feb. 1, 2016.

2010 Mathematics Subject Classification. Primary 46N10; Secondary 26A48, 26A51, 26A46, 26D05, 26D07, 26D10, 26D15, 34L30, 41A10, 46A55, 49K30, 49M29, 52A07, 52A41, 60E15, 90C25, 90C46.

Keywords. dual cones, multiply monotone functions, generalized moments, stochastic orders, probability inequalities. 


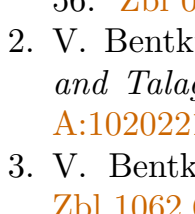

ANALYSIS
Banach J. Math. Anal. 10 (2016), no. 4, 864-897

http://dx.doi.org/10.1215/17358787-3649788

ISSN: $1735-8787$ (electronic)

http://projecteuclid.org/bjma

\title{
CONVEX CONES OF GENERALIZED MULTIPLY MONOTONE FUNCTIONS AND THE DUAL CONES
}

\author{
IOSIF PINELIS
}

Communicated by D. Yang

\begin{abstract}
Let $n$ and $k$ be nonnegative integers such that $1 \leq k \leq n+1$. The convex cone $\mathscr{F}_{+}^{k: n}$ of all functions $f$ on an arbitrary interval $I \subseteq \mathbb{R}$ whose derivatives $f^{(j)}$ of orders $j=k-1, \ldots, n$ are nondecreasing is characterized. A simple description of the convex cone dual to $\mathscr{F}_{+}^{k: n}$ is given. In particular, these results are useful in, and were motivated by, applications in probability. In fact, the results are obtained in a more general setting with certain generalized derivatives of $f$ of the $j$ th order in place of $f^{(j)}$. Somewhat similar results were previously obtained, in terms of Tchebycheff-Markov systems, in the case when the left endpoint of the interval $I$ is finite, with certain additional integrability conditions; such conditions fail to hold in the mentioned applications. Development of substantially new methods was needed to overcome the difficulties.
\end{abstract}

\section{INTRODUCTION}

In applications in probability (see, e.g., [11], [5], [6], [20], [21], [3], [26], [23], [25], and references therein), one is concerned with stochastic domination, defined by a formula of the form

$$
X \succcurlyeq Y \stackrel{\mathscr{F}}{\Longleftrightarrow} X \succcurlyeq Y(\bmod \mathscr{F}) \Longleftrightarrow \mathrm{def} f(X) \geq \mathrm{E} f(Y) \text { for all } f \in \mathscr{F},
$$

Copyright 2016 by the Tusi Mathematical Research Group.

Received Jul. 22, 2015; Accepted Feb. 1, 2016.

2010 Mathematics Subject Classification. Primary 46N10; Secondary 26A48, 26A51, 26A46, 26D05, 26D07, 26D10, 26D15, 34L30, 41A10, 46A55, 49K30, 49M29, 52A07, 52A41, 60E15, 90C25, 90C46.

Keywords. dual cones, multiply monotone functions, generalized moments, stochastic orders, probability inequalities. 


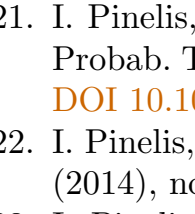

ANALYSIS
Banach J. Math. Anal. 10 (2016), no. 4, 864-897

http://dx.doi.org/10.1215/17358787-3649788

ISSN: $1735-8787$ (electronic)

http://projecteuclid.org/bjma

\title{
CONVEX CONES OF GENERALIZED MULTIPLY MONOTONE FUNCTIONS AND THE DUAL CONES
}

\author{
IOSIF PINELIS
}

Communicated by D. Yang

\begin{abstract}
Let $n$ and $k$ be nonnegative integers such that $1 \leq k \leq n+1$. The convex cone $\mathscr{F}_{+}^{k: n}$ of all functions $f$ on an arbitrary interval $I \subseteq \mathbb{R}$ whose derivatives $f^{(j)}$ of orders $j=k-1, \ldots, n$ are nondecreasing is characterized. A simple description of the convex cone dual to $\mathscr{F}_{+}^{k: n}$ is given. In particular, these results are useful in, and were motivated by, applications in probability. In fact, the results are obtained in a more general setting with certain generalized derivatives of $f$ of the $j$ th order in place of $f^{(j)}$. Somewhat similar results were previously obtained, in terms of Tchebycheff-Markov systems, in the case when the left endpoint of the interval $I$ is finite, with certain additional integrability conditions; such conditions fail to hold in the mentioned applications. Development of substantially new methods was needed to overcome the difficulties.
\end{abstract}

\section{INTRODUCTION}

In applications in probability (see, e.g., [11], [5], [6], [20], [21], [3], [26], [23], [25], and references therein), one is concerned with stochastic domination, defined by a formula of the form

$$
X \succcurlyeq Y \stackrel{\mathscr{F}}{\Longleftrightarrow} X \succcurlyeq Y(\bmod \mathscr{F}) \Longleftrightarrow \mathrm{def} f(X) \geq \mathrm{E} f(Y) \text { for all } f \in \mathscr{F},
$$

Copyright 2016 by the Tusi Mathematical Research Group.

Received Jul. 22, 2015; Accepted Feb. 1, 2016.

2010 Mathematics Subject Classification. Primary 46N10; Secondary 26A48, 26A51, 26A46, 26D05, 26D07, 26D10, 26D15, 34L30, 41A10, 46A55, 49K30, 49M29, 52A07, 52A41, 60E15, 90C25, 90C46.

Keywords. dual cones, multiply monotone functions, generalized moments, stochastic orders, probability inequalities. 